\title{
Design of a High-Bandwidth Tripod Scanner for High Speed Atomic Force Microscopy
}

\author{
Chen Yang, ${ }^{1,2} \mathrm{Jihong}_{\mathrm{H}}$ an, ${ }^{1}$ Maja Dukic, ${ }^{2}$ Nahid Hosseini, ${ }^{2} \mathrm{JIE}_{\mathrm{Z}} \mathrm{ZhaO},{ }^{1}$ and Georg E. Fantner ${ }^{2}$ \\ ${ }^{1}$ State Key Laboratory of Robotics and Systems, Harbin Institute of Technology, Harbin, China \\ ${ }^{2}$ Laboratory for Bio and Nano Instrumentation, Interfaculty Institute of Bioengineering, École Polytechnique Fédérale \\ de Lausanne, Lausanne, Switzerland
}

\begin{abstract}
Summary: Tip-scanning high-speed atomic force microscopes (HS-AFMs) have several advantages over their sample-scanning counterparts. Firstly, they can be used on samples of almost arbitrary size since the high imaging bandwidth of the system is immune to the added mass of the sample and its holder. Depending on their layouts, they also enable the use of several tipscanning HS-AFMs in combination. However, the need for tracking the cantilever with the readout laser makes designing tip-scanning HS-AFMs difficult. This often results in a reduced resonance frequency of the HS-AFM scanner, or a complex and large set of precision flexures. Here, we present a compact, simple HS-AFM designed for integrating the self-sensing cantilever into the tipscanning configuration, so that the difficulty of tracking small cantilever by laser beam is avoided. The position of cantilever is placed to the end of whole structure, hence making the optical viewing of the cantilever possible. As the core component of proposed system, a high bandwidth tripod scanner is designed, with a scan size of $5.8 \mu \mathrm{m} \times 5.8 \mu \mathrm{m}$ and a vertical travel range of $5.9 \mu \mathrm{m}$. The hysteresis of the piezoactuators in X- and $\mathrm{Y}$-axes are linearized using input shaping technique. To reduce in-plane crosstalk and vibration-related
\end{abstract}

Contract grant sponsor: European Union FP-7 program Eurostars E! 8213; Contract grant sponsor: European Union FP7/2007-2013/ERC; Contract grant number: 307338-NaMic; Contract grant sponsor: Swiss National Science Foundation; Contract grant number: 205320_152675; Contract grant sponsor: China Scholarship Council; Contract grant number: 201306120115

Address for reprints: Jie Zhao, State Key Laboratory of Robotics and Systems, Harbin Institute of Technology, Harbin, China.

E-mail: jzhao@hit.edu.cn

Address for reprints: Georg E. Fantner, Laboratory for Bio and Nano Instrumentation, Interfaculty Institute of Bioengineering, École Polytechnique Fédérale de Lausanne, Lausanne, Switzerland.

E-mail: georg.fantner@epfl.ch

Received 3 May 2016; Accepted with revision 27 June 2016

DOI: $10.1002 /$ sca. 21338

Published online 2 August 2016 in Wiley Online Library

(wileyonlinelibrary.com). dynamics, we implement both filters and compensators on a field programmable analog array. Based on these, images with $512 \times 256$ pixels are successfully obtained at scan rates up to 1024 lines/s, corresponding to a $4 \mathrm{~mm} / \mathrm{stip}$ velocity. SCANNING 38:889-900, 2016. (C) 2016 Wiley Periodicals, Inc.

Key words: high speed atomic force microscopy, tipscanning; tripod scanner, compliant mechanism, selfsensing cantilevers

\section{Introduction}

Thorough understanding of surface phenomena happening at the nanoscale is a key issue in many research fields. A series of exciting discoveries have been reported recently,benefiting from the advances of high speed atomic force microscopy (HS-AFM). For example, the walking behaviours of a myosin $\mathrm{V}$ molecule was directly visualized, which provided insight into the nature of this molecular motor (Kodera et al., 2010); by characterizing the diffusion dynamics of the outer membrane protein $\mathrm{F}$, connection between structures of individual proteins and dynamics of supramolecular membranes was revealed (Casuso et al., 2012); and successful implementation of fast AFM imaging on Escherichia coli revealed several phases of the killing process of an anti-microbial peptide (Fantner et al., 2010). For materials analysis, studies of photoresist dissolution supported several previous assumptions and helped the design of photoresist for next generation applications (Itani and Santillan, 2010). Indeed, many of the efforts aim to capture dynamic process at the nanoscale are put into atomic force microscopy, for the reason that it is the only tool can be utilized in air, vacuum, and liquid while still keeping the nanometer resolution.

AFMs can be operated in contact or dynamic mode. Although, details of the dynamic mode are out of the scope of this paper, extensive studies have been presented (Hansma et al., '94; Sulchek et al., 2000; 
Sulchek et al., 2002; Ando et al., 2008). The principle of AFMs operated in (constant force) contact mode is to keep the deflection of micromechanical cantilever constant when raster scanning the sample surface in the X-Y plane. The constant deflection is achieved by using a feedback controller which adapts the cantileversample distance to track the surface change. Then the output of feedback controller is recorded and used for topography reconstruction.

In order to improve the time resolution of AFM, critical components such as cantilever (Fantner et al., 2006; Ando et al., 2008; Adams et al., 2015), scanner (Humphris et al., 2005; Fukuma et al., 2008; Braunsmann and Schäffer, 2010), feedback controller (Kodera et al., 2006; Kuiper and Schitter, 2012), high voltage amplifier (Fleming and Leang, 2008; Fleming, 2009), and data acquisition system (Fantner et al., 2005) need to be redesigned. The limitation from the scanner is that the low first resonance mode of the $\mathrm{X}$ - and Y-axes prohibit higher scan rates. For example, conventional piezo-tube AFM scanners have a lateral resonance frequency around $1 \mathrm{kHz}$, hence the maximum scan rates that can be achieved is only around $10 \mathrm{~Hz}$ (1\% of resonance frequency) (Croft et al., '99), when driven by a triangular waveform. Non-raster scan paths such as spiral, cycolid and Lissajous trajectories do help to speed up imaging process (Mahmood and Moheimani, 2009; Yong et al., 2010; Tuma et al., 2012); however, the sinusoidal nature of which increases computational complexity and leads to images with uneven-distributed resolution. While the low X-and Y-axes resonances can be compensated with feedforward input shaping (Leang and Devasia, 2007; Schitter et al., 2008), the low Z-resonance does impose severe limitation on the maximum achievable tracking speed. To solve this problem and hence improve the dynamic performance of the AFM system, several flexure-based high bandwidth scanner have been proposed (Kindt et al., 2004; Schitter et al., 2007; Kenton and Leang, 2012; Yong et al., 2013), which are driven by piezo-stack actuators as well as hybrid systems consisting of piezo-tube and stack actuators (Nievergelt et al., 2015).

Conceptually, AFMs can be implmented in two main configurations, namely sample-scanning mode and tip-scanning mode. In sample-scanning configuration, the sample is carried by the scanner. As the mechanical bandwidth of the scanner is sensitive to the added mass, the weight and volume of the sample need to be highly restricted as not to dramatically limit the imaging bandwidth. Furthermore, when changing the mass of samples, the resonance frequency of the Z-scanner will shift, therefore, making control schemes involving system identification impractical, since the system identification procedure has to be repeated for every experiment. On the other hand, in tip-scanning configuration, the cantilever is moved by the scanner in $\mathrm{X}-, \mathrm{Y}-$, and $\mathrm{Z}$-direcitons, making the Z-scanner bandwidth independent of the sample. A combination of these two schemes is also possible. Nevertheless, most of existed high speed AFM systems were not designed for tip-scanning configuration, since in this case the prevalently used optical detection system for cantilever deflection requires tracking of cantilever's motion in the XY plane by the laser beam. Integrating the laser, position sensitive detector and lenses into the scanner is a possible solution; however, the mechanical bandwidth will be negatively impacted by the added mass.

In this paper, a high speed tip-scanning AFM system is presented, the core of which is a flexure guided high bandwidth tripod scanner. The tripod configuration is chosen since the scanner can be designed very stiff in this manner: three piezoactuators support each other which makes the whole structure equivalently stiff in each driving direction, hence removing the undesired tampoline mode that generally appears in other types of flexure guided piezo-stack based AFM scanners. Additionally, by using selfsensing cantilevers, detecting optics are completely eliminated which dramatically simplifies the design and daily use. Without the interference of optics, the cantilever can be located at the front end of the whole structure, so that high magnification optical viewing can be easily integrated into the system. The standalone and open nature of presented system allows the operation of multiple independent HS-AFMs in close proximity.

\section{Method}

\section{Tripod Scanner Configuration Design}

The basic configuration of a tip-scanning tripod scanner is shown in Figure 1(a), where three piezostacks are arranged perpendicularly to each other and coupled together at one point. The detailed design is determined by balancing several key characteristics such as motion coupling, travel range, and dynamic performance. Figure 1(b-d) illustrate three different design schemes for a single axis, the advantages and drawbacks of each are discussed as follows. In the first scheme, the rotational flexure, which aims to reduce motion coupling between the driving axes, is placed between the stationary side of the piezo-stack and the fixed support. One great virtue of this configuration is that the distance $D_{1}$ from the point where the cantilever is located to the rotational centre is maximized, so that motion coupling, especially $\mathrm{X}-\mathrm{Y}$ coupling, can be reduced significantly. However, the bending moment around the flexure point generated by the weight of cantilever holder $\left(M_{1}=D_{1} \times W_{l}\right)$ and piezo-stack $\left(M_{2}=D_{2} \times W_{2}\right)$ are also maximized which 


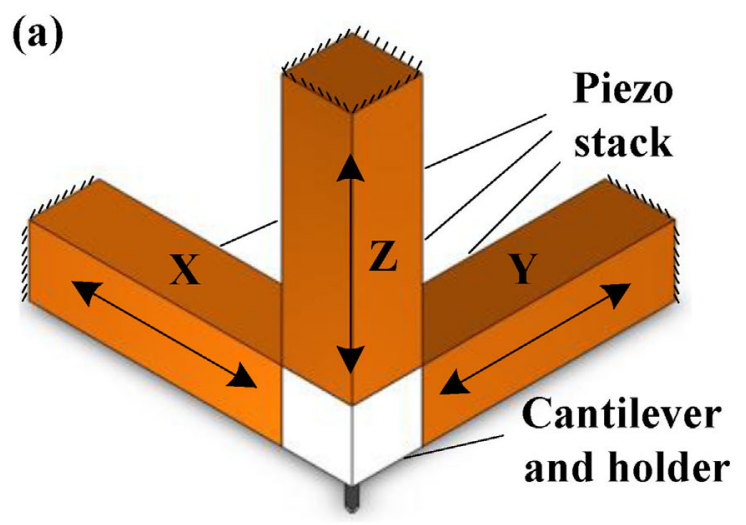

(b)

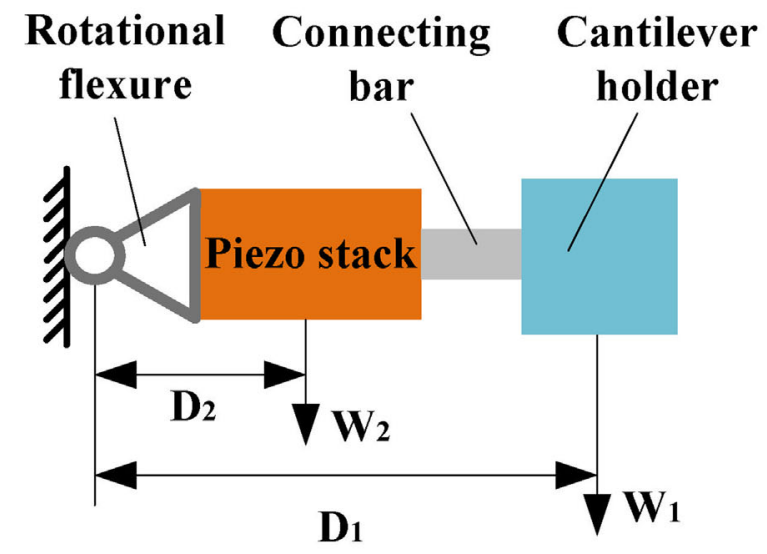

Fig 1. Comparison of designs for high speed tripod scanner.

slows down the dynamic response of the structure. The second scheme makes a compromise by arranging the rotational flexure in the middle between the moving side of the piezo and the cantilever holder, while keeping the stationary side of the piezo-stack rigidly attached to the fixed support. The advantage is that the bending moment around the flexure point is reduced, but at the cost of increased motion coupling. However, this coupling can be compensated by manipulating the driving signal, which will be discussed later in detail. As the bending mode of the piezoactuator is still the decisive mode for its first resonance, Figure 1(d) provides an alternative solution using a planar flexure to reduce the influence of this mode. The requirements for such a flexure are that its lateral stiffness needs to be high enough while keeping the out-of-plane stiffness at a low level. Figure 2(a) shows an assembled planar flexure that satisfies these requirements, the design of which is inspired by diaphragm flexures. The flexures are manufactured using electrical discharge machining (EDM) out of Aluminum alloy 7075 , because of its high Young's modulusdensity ratio.
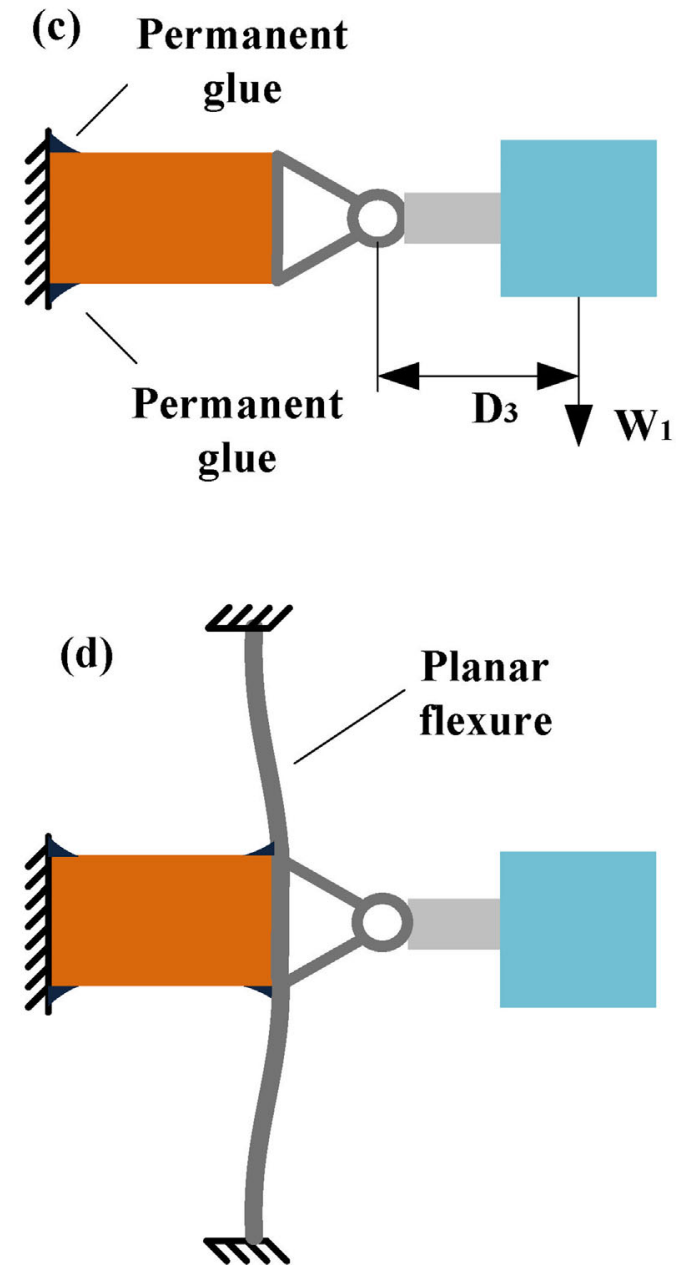

\section{Flexure Design and Simulation}

The planar flexure is designed to improve the lateral stiffness of the piezoactuator without losing much range. As a rule of thumb, this means the total stiffness of the flexures (including the stiffness of both planar and rotational flexures) needs to follow the relationship (Kenton and Leang, 2012; Yong et al., 2012):

$$
k_{\text {flexure }} \leq k_{\text {piezo }} / 10
$$

The actual motion range of the scanner $\delta$ is

$$
\delta=\frac{k_{\text {piezo }}}{k_{\text {piezo }}+k_{\text {flexure }}} \delta_{0} \geq 0.9 \delta_{0}
$$

where $\delta_{0}$ is the nominal travel range of the piezoactuator.

Here Castigliano's second theorem is used to guide the stiffness analysis. Due to the symmetric essence, only a quater of the whole flexure is taken, the dimensions and analytical model of which are shown in Figure 2(c) and (d). As the flexure is 
(a)

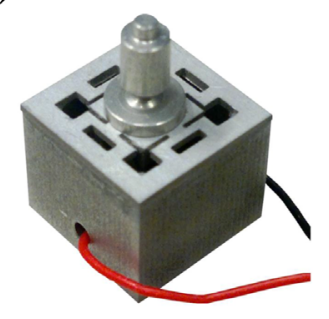

(b)

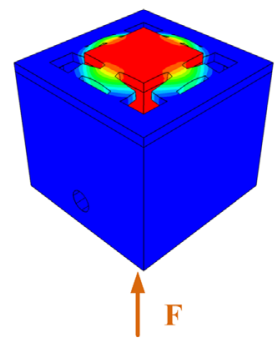

(c)

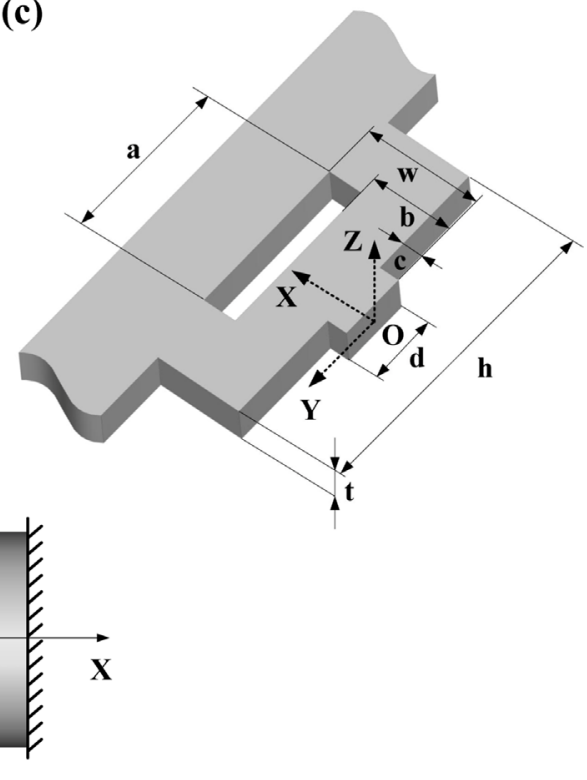

Fig 2. (a) Piezoactuator with proposed planar and rotational flexure; (b) Finite-element stiffness simulation; (c) Schematic of the planar flexure, the dimensionsof which are as follows: $\mathrm{a}=2.4, \mathrm{~b}=1.2, \mathrm{c}=0.3, \mathrm{~d}=1, \mathrm{~h}=4.4, \mathrm{t}=0.6$, and $\mathrm{w}=1.9 \mathrm{~mm}$; (d) Analytical model of the planar flxure stiffness; and (e) Analytical model of rotational flexure stiffness, in which $\mathrm{r}=0.8,1=3.4$, and $\mathrm{D}=3 \mathrm{~mm}$.

subjected to a fixed-guided boundary condition, loads of $M_{y}$ and $F_{z}$ actually act on the free end of the flexure. The coordinate system is constructed at the same position to simplify the subsequent calculation, without loss of generality. The resulting strain energy $U$ is:

$$
U=\int_{0}^{w}\left(\frac{\left(F_{z} x+M_{y}\right)^{2}}{2 E I(x)}+\frac{\alpha F_{z}^{2}}{2 G A(x)}\right) d x
$$

where $E$ is the Young's modulus, $G=E / 2(1+v)$ is the shear modulus, $\alpha$ is a shape-related coefficient (for rectangular cross section $\alpha=1.2), I(x)$, and $A(x)$ are the second moment of inertia and the area of cross section, respectively. For the given shape of the flexure, these are:

$$
I(x)=\left\{\begin{array}{cc}
I_{1}=\frac{d t^{3}}{12} & x \in[0, c] \\
I_{2}=\frac{h t^{3}}{12} & x \in[c, b] \\
I_{3}=\frac{t^{3}(h-a)}{12} & x \in[b, w]
\end{array}\right.
$$

and

$$
A(x)=\left\{\begin{array}{cc}
A_{1}=d t & x \in[0, c] \\
A_{2}=h t & x \in[c, b] \\
A_{3}=t(h-a) & x \in[b, w]
\end{array}\right.
$$

Because of the fixed-guided boundary condition, the rotational angle of the beam at origin equals to zero, i.e. $\left.\theta_{y}\right|_{x=0}=0$. According to Castigliano's second theorem, $\theta_{y}$ can be written as:

$$
\theta_{y}=\frac{\partial U}{\partial M_{y}}
$$

Therefore, the relationship between $M_{y}$ and $F_{z}$ is:

$$
M_{y}=-\frac{\frac{c^{2}}{I_{1}}+\frac{b^{2}-c^{2}}{I_{2}}+\frac{w^{2}-b^{2}}{I_{3}}}{2\left(\frac{c}{I_{1}}+\frac{b-c}{I_{2}}+\frac{w-b}{I_{3}}\right)} F_{z}=-S \cdot F_{z}
$$

To calculate the stiffness of the planar flexure, we can take a similar procedure, the resultant deflection of the cantilever beam is:

$$
u_{z}=\frac{\partial U}{\partial F_{z}}=\int_{0}^{w}\left(\frac{\left(F_{z} x+M_{y}\right) \cdot x}{E I(x)}+\frac{\alpha F_{z}}{G A(x)}\right) d x
$$

By substituting (6) into (7), we have:

$$
k_{z}=\frac{F_{z}}{u_{z}}=\left[\int_{0}^{w}\left(\frac{x(x-S)}{E I(x)}+\frac{\alpha}{G A(x)}\right) d x\right]^{-1}
$$

The calculated out-of-plane stiffness of the planar flexure is $8.25 \mathrm{~N} / \mu \mathrm{m}$. Finite element analysis (FEA) was also implemented based on ABAQUS (Dassault Systems, France). Figure 2(b) illustrates the result of an evendistributed load applied on the bottom surface of the 
piezoactuator. The ratio between the applied load and resultant displacement provides the flexure stiffness of $6.02 \mathrm{~N} / \mu \mathrm{m}$.

The circular flexures aimed at reducing the motion couplings are also carefully designed. The stiffness analysis is conducted in a similar way by using fixed-free boundary conditon (as shown in Figure 2(e)). The stiffness of the rotational flexure can be written as:

$$
k=\left[\int_{0}^{l} \frac{x^{2} d x}{E I(x)}+\int_{l}^{l+2 r} \frac{\alpha d x}{G A(x)}\right]^{-1}
$$

where

$$
I(x)=\left\{\begin{array}{cc}
\frac{\pi D^{4}}{64} & x \in[0, l] \\
\frac{\pi}{64}\left[D-2 \sqrt{r^{2}-(x-r)^{2}}\right]^{4} & x \in[l, l+2 r]
\end{array}\right.
$$

$$
A(x)=\left\{\begin{array}{cc}
\frac{\pi D^{2}}{4} & x \in[0, l] \\
\frac{\pi}{4}\left[D-2 \sqrt{r^{2}-(x-r)^{2}}\right]^{2} & x \in[l, l+2 r]
\end{array}\right.
$$

The calculated stiffness is only $0.81 \mathrm{~N} / \mu \mathrm{m}$ so that the total stiffness introduced by all of the flexures is less than $10 \%$ of the piezoactuator's stiffness $(100 \mathrm{~N} / \mu \mathrm{m}$ for P-885.11, PI, Germany).

Based on these considerations and results, the scanner is designed and shown as Figure 3(b1). To further evaluate the impact of planar flexure on scanner resonance, a FEA results comparison is presented in Figure 3. (Due to the symmetric nature, $\mathrm{X}$ - and $\mathrm{Y}$-axes resonance should be the same, therefore we use lateral resonance for simplicity.) The simulation results indicate that by using planar flexure, the lateral resonance increases from 14.1 to $24.0 \mathrm{kHz}$, while the vertical resonance reaches $28.2 \mathrm{kHz}$.

\section{Dynamic Response Characterization and Filter Implementation}

The coupling nature of the tripod scanner makes both the static and dynamic characterization necessary. Here we use $\operatorname{Gij}(i, j \in\{x, y, z\})$ to represent the dynamic response of $j$-axis when driving $i$-axis. Figure 4 plots the measured dominant resonance frequency of each axis $(G x x, G y y$, and $G z z)$ and the coupling dynamics (Gxy, $G x z, G y x$, Gyz, Gzx, and Gzy). These were measured by sweeping the actuation frequency. The sweeping signal was obtained from the reference output of a lock-in amplifier (e-LockIn 204/2, Anfatec, Germany), then amplified by a high voltage piezo amplifier (Techproject, Austria, which can provide 8A of current (a1)

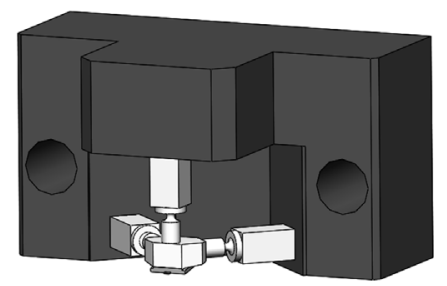

Tripod scanner without planar flexure

(b1)

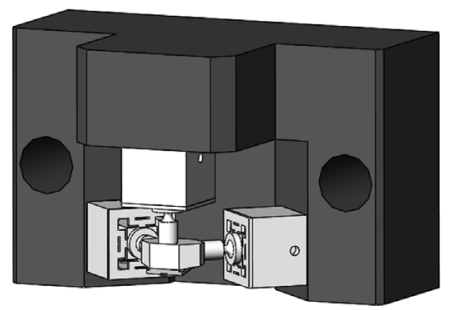

Tripod scanner with planar flexure (a2)

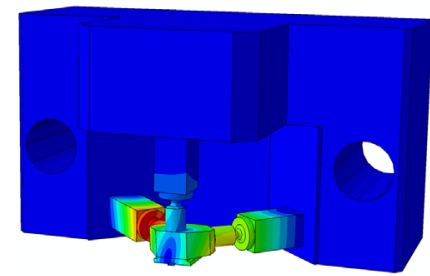

Lateral resonance

$\mathbf{f}=14.1 \mathrm{kHz}$

(b2)

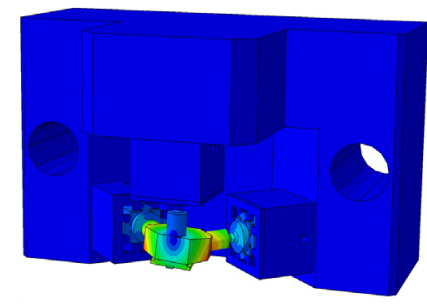

Lateral resonance $\mathbf{f}=\mathbf{2 4 . 0} \mathrm{kHz}$ (a3)

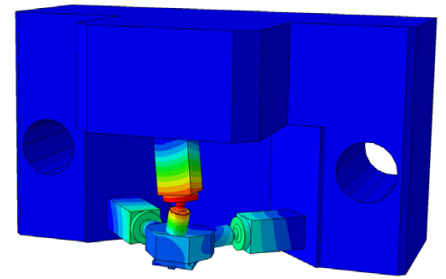

Vertical resonance $\mathrm{f}=18.4 \mathrm{kHz}$

(b3)

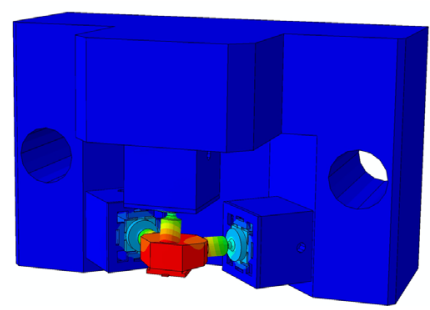

Vertical resonance $\mathbf{f}=28.2 \mathrm{kHz}$

Fig 3. (a1) and (b1) are mechanical configurations of the scanner with and without planar flexure. (a2), (a3), (b2), and (b3) are lateral and vertical resonances of each configuration. 

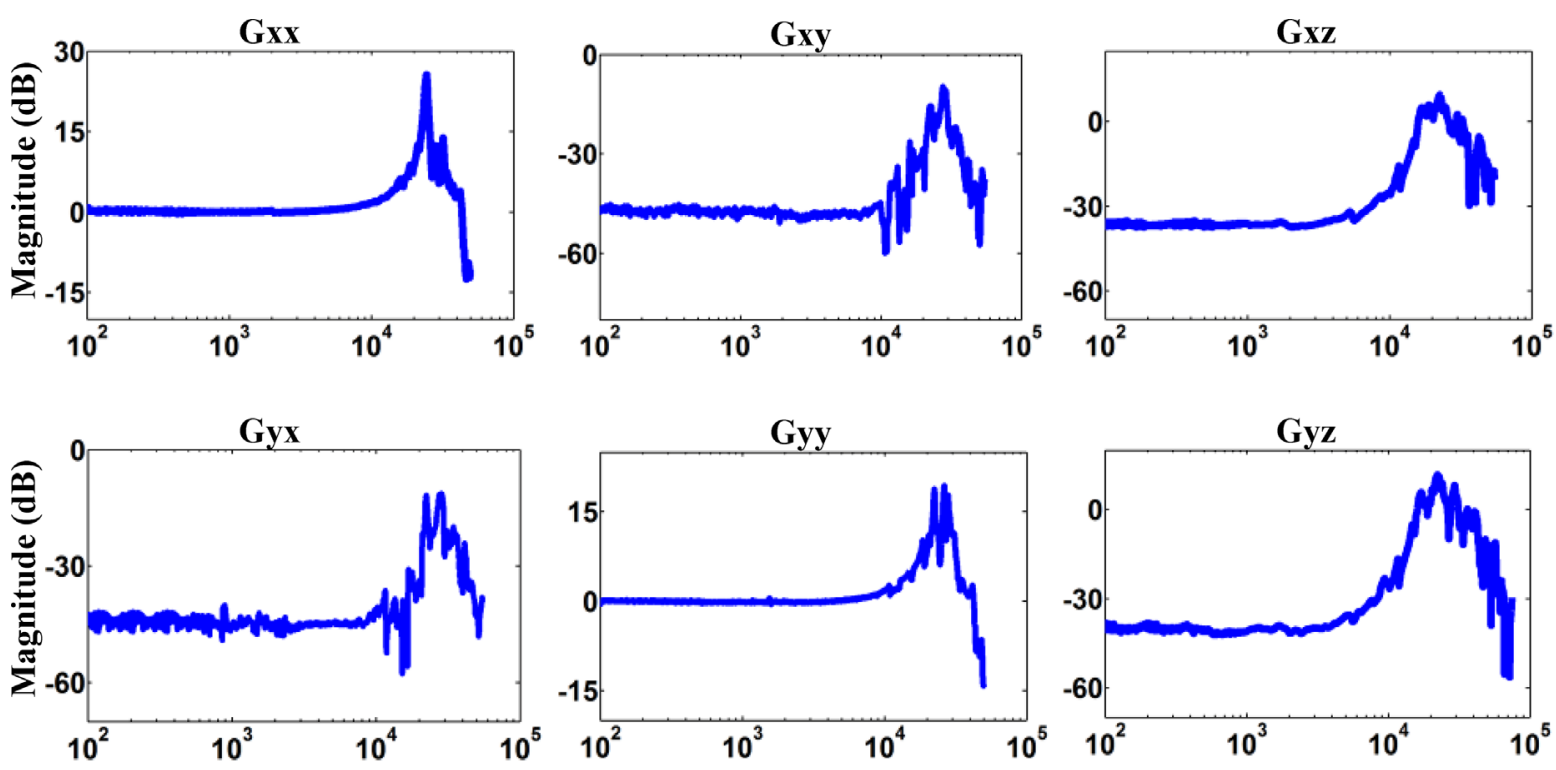

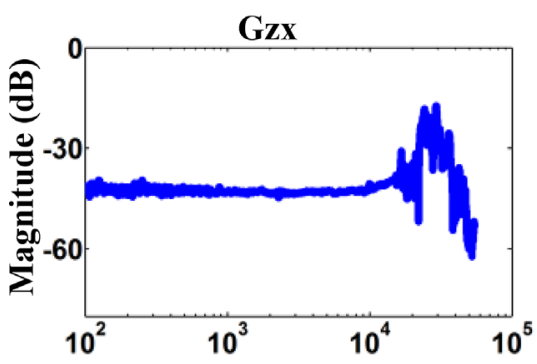

Frequency $(\mathrm{Hz})$

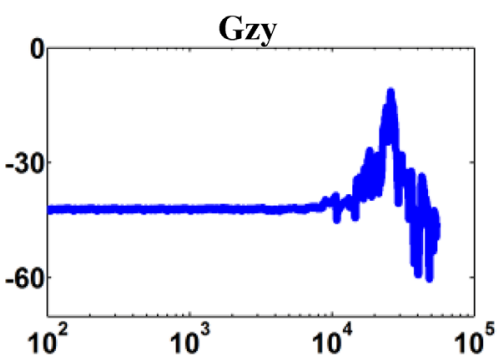

Frequency $(\mathrm{Hz})$

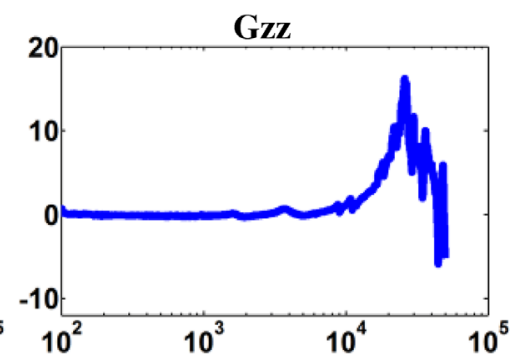

Frequency $(\mathbf{H z})$

Fig 4. Measured frequency responses of the scanner.

at $150 \mathrm{~V}$ and more than $40 \mathrm{kHz}$ bandwidth for the used $0.6 \mu \mathrm{F}$ piezoactuator). The collected sensor signal was routed to the signal input of lock-in amplifier for analysis. A laser doppler vibrometer (Polytec OFV-512, Germany) was used for the $\mathrm{X}$ - and $\mathrm{Y}$-axes displacement measurement. To capture the $\mathrm{Z}$-axis related dynamic responses, a self-sensing cantilever (PRS-L70-F350, SCL-Sensor. Tech, Austria) with $520 \mathrm{kHz}$ resonance was used to keep in contact with the substrate surface, then collecting the signal from a home-built cantilever readout board (with $1 \mathrm{MHz}$ bandwidth). Figure 4 indicates that the first eigenmodes for $\mathrm{X}-, \mathrm{Y}-$, and Z-axes are 24.5, 22.3, and $26.7 \mathrm{kHz}$, respectively.

In order to achieve high speed imaging, special attentions needed to be paid to both Z-Z and X-Z (or $\mathrm{Y}-\mathrm{Z}$ ) dynamic responses, since the former limits the bandwidth of the feedback loop while the latter practically restricts the line-scan rate to approximately $1 \%$ of the resonance frequency. By designing a proper filter or compensator, the effect of undesired dynamics can be significantly reduced. The proposed solution is implemented based on Field Programmable Analog Arrays (FPAA) development board (AN231K04QUAD4, Anadigm) (Schitter and Phan, 2008; Yong et al., 2013), the schematic of which is shown in Figure 5.
Besides cost-efficiency, one apparent advantage of FPAA is that filters or compensators can be easily implemented and modified in a drag-and-drop way, while keeping typical signal bandwidth up to $2 \mathrm{MHz}$.

The adopted development board contains four chips (namely FPAA1-4) and a set of signal convertors, so that single ended signals can be converted to their differential forms and vice versa. FPAA1 serves as a high-bandwidth PI controller, where the deflection signal of the cantilever is applied to 1-2 leads differentially and the setpoint signal is set by using a digitally controlled, onboard DC signal. FPAA2 filters the output of PI controller to reduce the influence of Z-resonance (by connecting 21-22 leads of FPAA1 to 1-2 leads of FPAA2). FPAA3 is adopted to damp X-axis dynamics, which is essential to achieve high imaging rate. After being filtered, the fast-axis driving signal (namely output signal of FPAA3) is connected to 15-16 leads of FPAA4 as one input, while another input is the slow-axis driving signal introduced via 1-2 leads of the same chip. FPAA4 is a compensator proposed to reduce the motion coupling between $\mathrm{X}$ and $\mathrm{Y}$ axes, details of which will be discussed in the following section. Arrows in Figure 5 indicate the signal flow before and after the FPAA. 


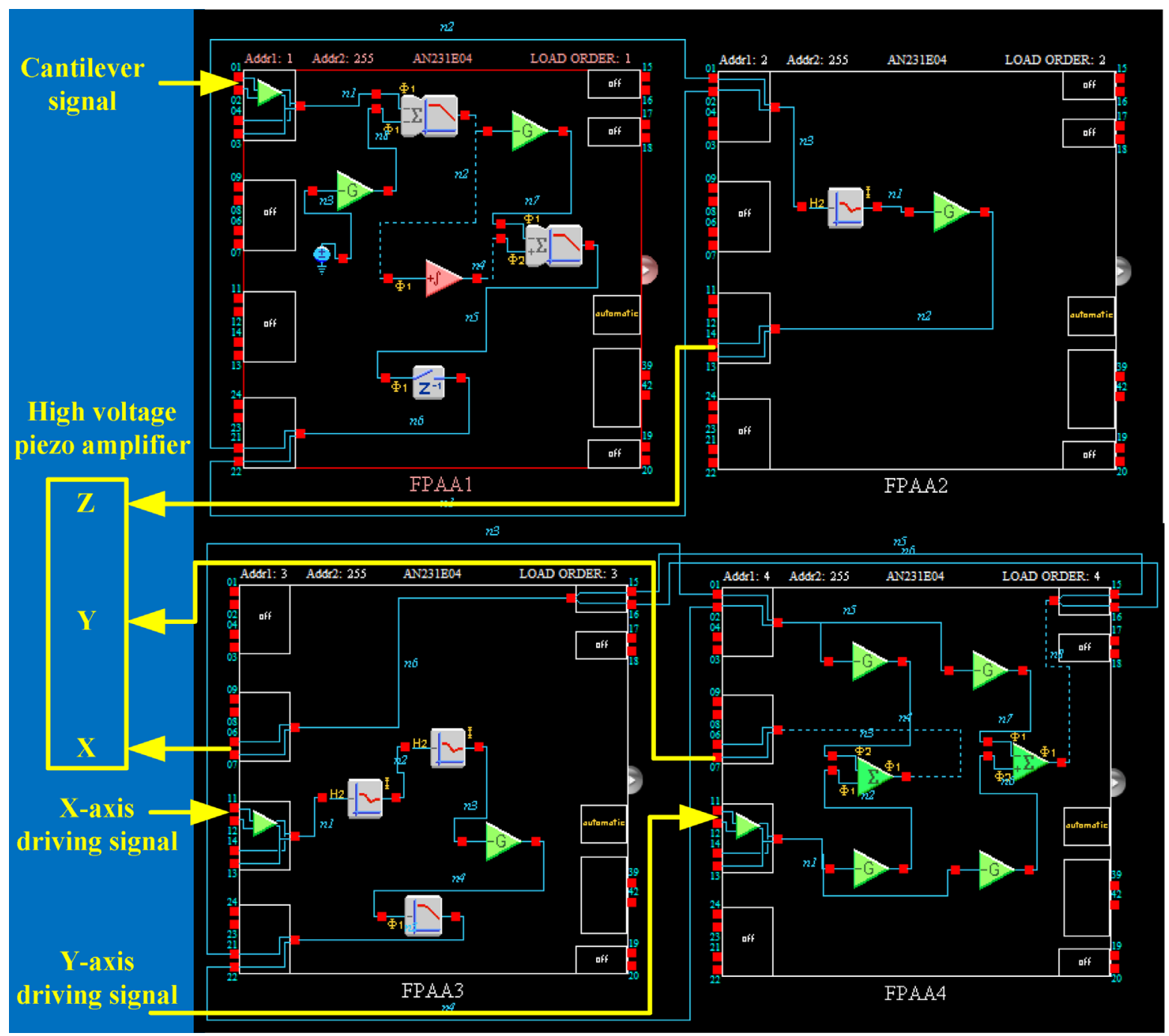

Fig 5. Control electronics implemented based on FPAA.

Different from model-based filter design method which generally involves curve-fitting and inversion steps, the proposed filter is designed in a more intuitive way. Zeros and poles of the filter are selected until the resonance peak of a given frequency response is flattened. These poles and zeros are then used as direct setting parameters for the FPAA board. This method is practically useful especially when there are more than one resonance peak appearing in a narrow frequency range, since in this case getting a mathematically accurate model is challenging.

As the result, a fourth-order filter aimed to damp the $\mathrm{X}-\mathrm{Z}$ coupling dynamics is obtained and can be written as:

$G f_{x-z}=0.164 \times \frac{s^{4}+6.307 \times 10^{4} s^{3}+3.79 \times 10^{10} s^{2}+1.079 \times 10^{15} s+3.08 \times 10^{20}}{s^{4}+3.807 \times 10^{5} s^{3}+4.78 \times 10^{10} s^{2}+2.31 \times 10^{15} s+5.04 \times 10^{19}}$

The performance of the filter is illustrated in Figure 6 (a). From the bode plot, one can see the amplitude response included more than one peak, by adopting the proposed higher order filter, unwanted coupling dynamics was substantially flattened. Although, a small fluctuation around $11 \mathrm{kHz}$ still exists, the real images did not show any evident distortion even when operating at very high scan rate.

A similar process holds for obtaining the second-order Z-axis filter, which is:

$$
G f_{z}=1.022 \times \frac{s^{2}+3.105 \times 10^{4} s+2.73 \times 10^{10}}{s^{2}+1.721 \times 10^{5} s+2.789 \times 10^{10}}
$$

The lightly damped poles were cancelled by designing proper complex zeros of the filter, leading to flattened frequency response, as shown in Figure 6(b).

\section{Experimental Setup}

The overview of the home-built high speed AFM system is given in Figure 7. By using input-shaping technique, the driving signal were generated and sent to 


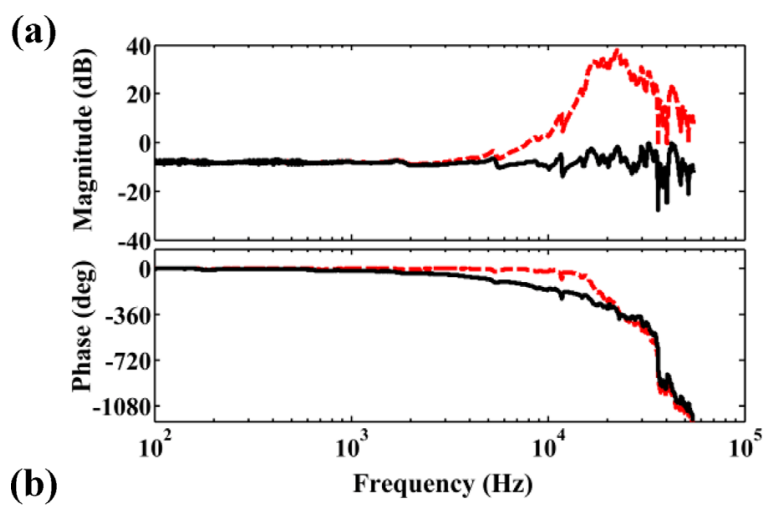

(b)

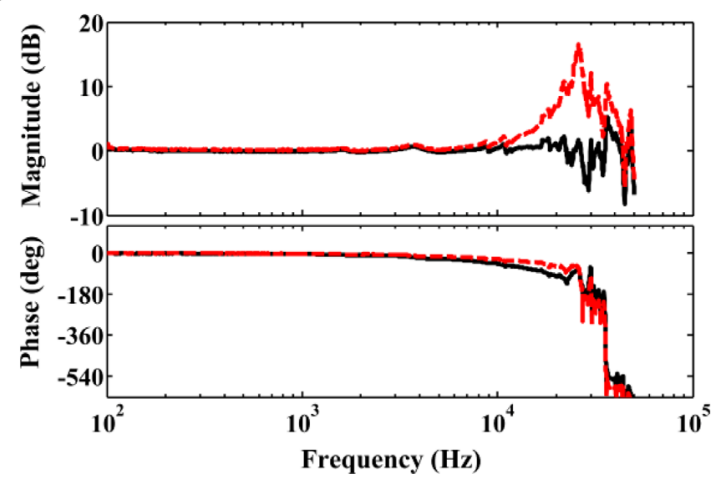

Fig 6. Filters designed for damping (a) X-Z coupling dynamics and (b) Z-scanner dynamics. The red and black curves are measured scanner dynamics before and after implementing the filters.

DAQ card (Daq2010, Adlink, Taiwan) through a LabVIEW-based software interface (Fantner et al., 2005). Then the card reproduced the signals according to the given scan rate and then passed them into FPAA development board (chip 4), where the coupling compensator was implemented. The outputs of the compensator were fed into a high voltage piezo amplifier to move the tripod scanner in both $\mathrm{X}$ - and Y-axes. Here the adopted self-sensing cantilever kept in contact with sample surface, interaction forces between the tip and sample were governed by the Z-feedback loop. The deflection signal of the cantilever was read by a home-built high-bandwidth readout board, which served as the input to the FPAA based PI controller (chip 1). Additionally, in order to tune $P$ and I gains online, a $\mathrm{C}++$ based interface was built. The controller output was delivered to both high voltage amplifier and DAQ card: the former aimed to control Z-scanner movement while the latter was used to generate the images.

\section{Results and Discussion}

\section{Motion Coupling Compensation}

Motion coupling between $\mathrm{X}$ - and Y-scanners exists since the trajectory of the cantilever holder is not exactly a straight line but part of circle with the center located at the position of the rotational flexure. As a result, when driving one axis at a given frequency, there is a small displacement induced in another direction with the same frequency. Additionally, coupling between in-plane and out-of-plane motion also exists for the same reason, resulting in a bowshape distorted image. However, by implementing image post-processing, the accurate sample topography can be recovered. So in this section, we only aim to reduce the coupled motion between X- and Y-scanners.

By applying $100 \mathrm{~V}$ peak-to-peak triangular wave input to $\mathrm{X}, \mathrm{Y}$, and $\mathrm{Z}$ piezoactuators, 5.8, 6.0, and 5.9 of travel range were obtained. The measurement was conducted using a laser interferometer with sensitivity of $0.5 \mathrm{~V} / \mu \mathrm{m}$. As the nominal travel range provided by

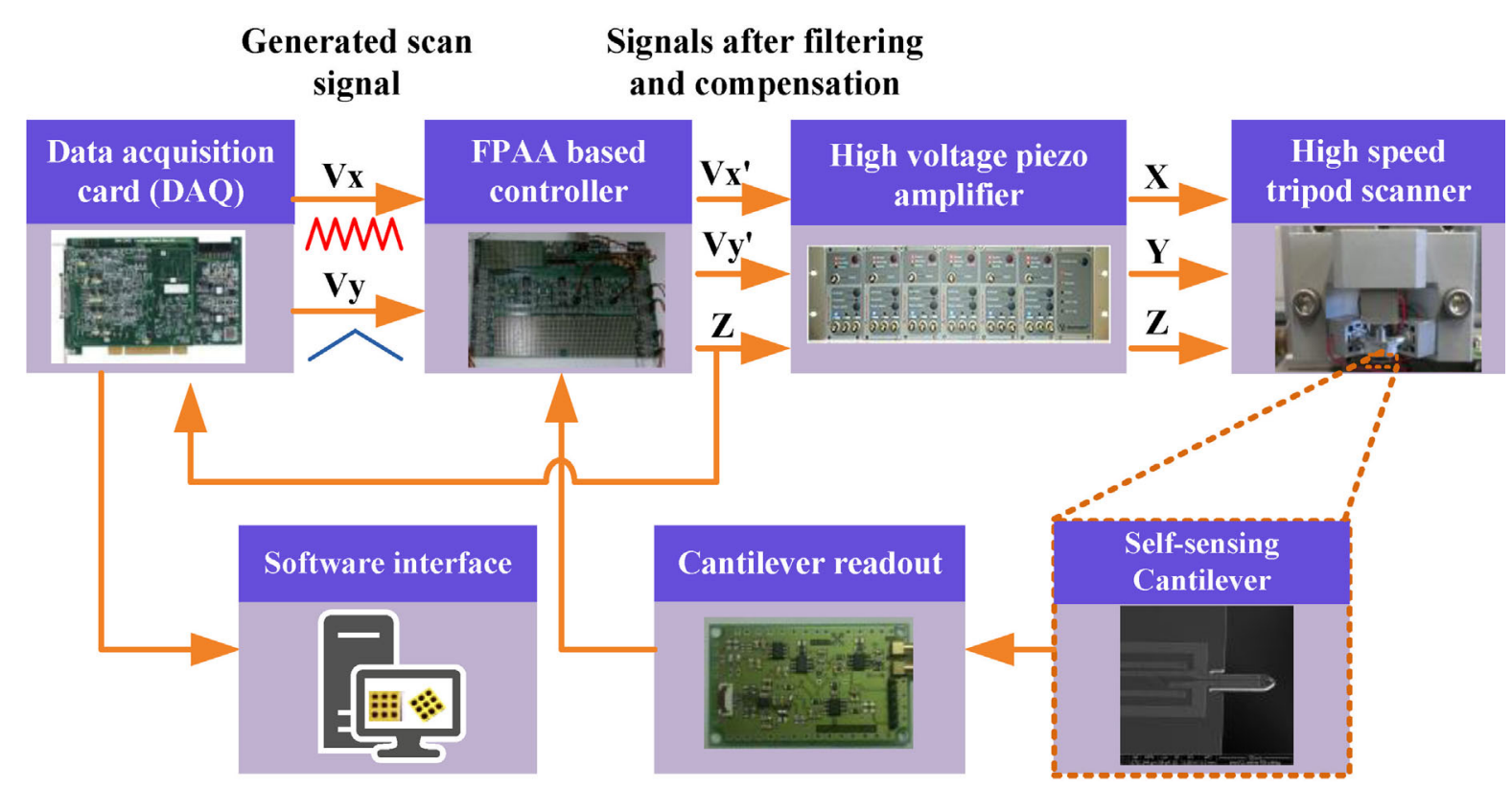

Fig 7. Overview of the home-built tip-scanning high speed AFM system. 
manufacturer is $6.5 \mu \mathrm{m}$, the actual travels reach to 89 , 92 , and $90 \%$ of the maximum, which further demonstrates the validity of flexures' design. Motion coupling characterizations of $\mathrm{Y}-\mathrm{X}, \mathrm{Z}-\mathrm{X}, \mathrm{X}-\mathrm{Y}$, and $\mathrm{Z}-\mathrm{Y}$ axes were conducted in the similar manner using the laser interferometer. The measured peak-to-peak coupling displacements were $0.24,0.13,0.26$, and $0.15 \mu \mathrm{m}$, respectively, which correspond to the coupling coefficients of $4.2 \%(-27.5 \mathrm{~dB}), 2.3 \%(-32.8 \mathrm{~dB})$, $4.4 \%-27.1 \mathrm{~dB})$, and $2.5 \%(-32 \mathrm{~dB})$. However, precise coupling measurements of $\mathrm{X}-\mathrm{Z}$ and $\mathrm{Y}-\mathrm{Z}$ axes were not conducted since the tilted angle of the cantilever holder made the procedure unfeasible.

As the coupled movement has the same frequency as driving signal, a practical way to reduce it is to take a small portion of driving signal then feed it into the passively moved axis. The principle can be expressed as:

$$
\left\{\begin{array}{l}
V_{x^{\prime}}=V_{x}-\alpha \cdot V_{y} \\
V_{y^{\prime}}=V_{y}-\beta \cdot V_{x}
\end{array}\right.
$$

in which $V_{x}$ and $V_{y}$ are driving signals obtained directly from data acquisition card (DAQ), while $V_{x^{\prime}}$ and $V_{y^{\prime}}$ are signals after compensation, parameters $\alpha$ and $\beta$ are tuned experimentally to minimize the coupled motion. Its performance is illustrated in Figure 8(a), where the
$\mathrm{X}$-axis was driven by a $128 \mathrm{~Hz}$ triangular wave at its full range (red line), and the coupled displacement of the Y-scanner (blue line) occurred in the opposite direction. By finely tuning $\beta$, the coupled displacement of the Y-axis was reduced significantly, as the black line shown. Figure 8(c) depicts the effect of the coupling in which the square patterns were distorted, resulting in sheared shapes. The sample used was a platinum-coated calibration grating with $1 \mu \mathrm{m}$ period and $100 \mathrm{~nm}$ depth (APCS-0001, Bruker). All the images were taken at a scan rate of $128 \mathrm{~Hz}$ and the resolution is $512 \times 256$ pixels, which corresponds to image rate of $0.5 \mathrm{~Hz}$.

\section{Hysteresis Compensation}

Piezoactuators exhibit highly nonlinear behavior in terms of hysteresis, especially when operating at high frequency, hence causing apparent image distortions. For compensating the hysteresis, either close-loop or open-loop methods can be used. Here, a cost-effective open-loop compensation method was adopted, based on the pre-measured hysteresis curve. The procedure of implementation was to fit the measured data by a fifthorder polynomial function first. Then the expected input signals were obtained by using ideally linear function to subtract this polynomial. Once getting the formula,

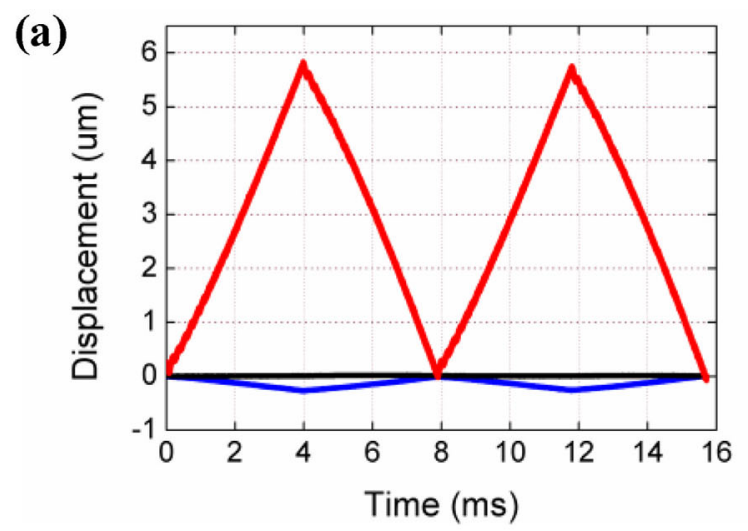

(c)

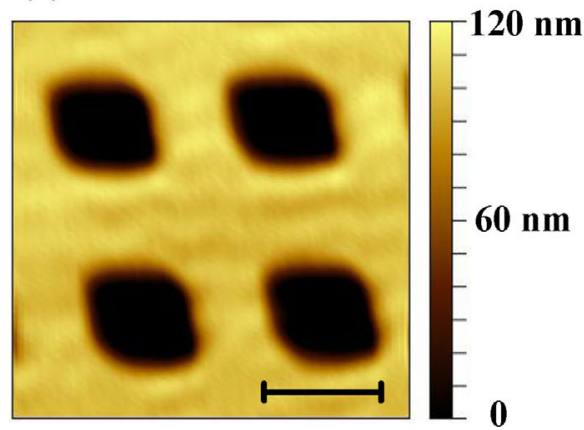

(b)

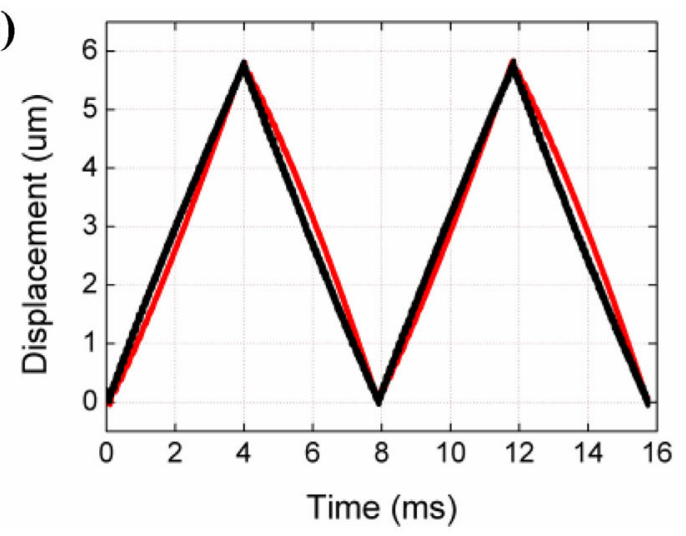

(e) (d)

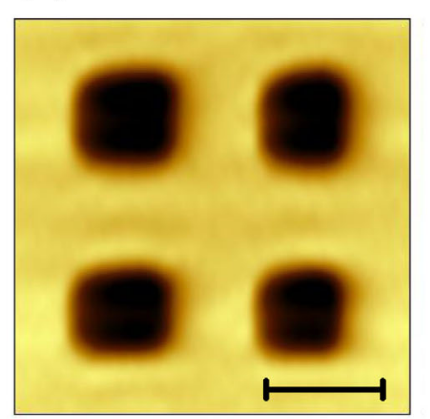

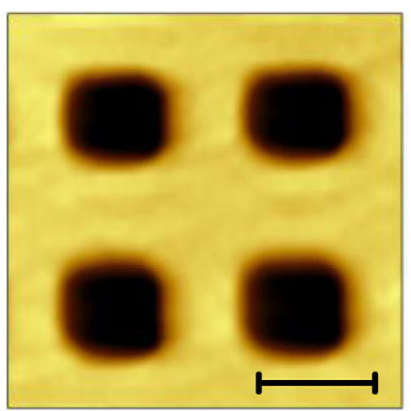

Fig 8. (a) Results of cross-coupling compensation. The red line is the motion of X-axis; blue line represents the corresponding coupled motion of Y-axis; black line gives the Y-axis motion after compensation. (b)Result of input-shaping hysteresis compensation. (c) and (d) shows the motion coupling and hysteresis of the scanner, respectively. (e) is the image after compensation. 


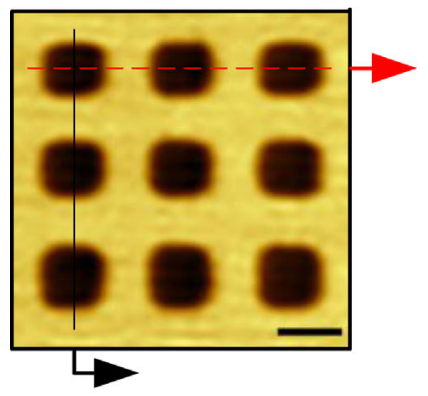

(a1)

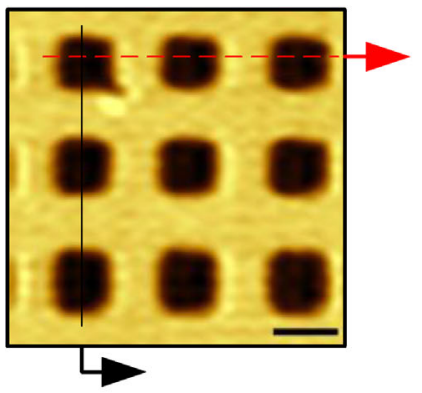

(b1)

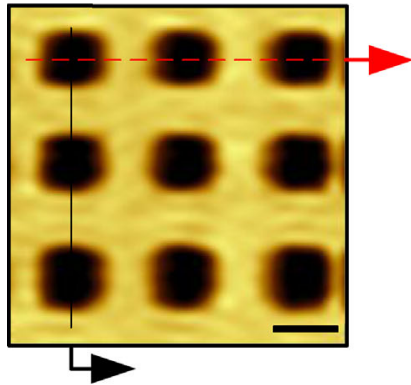

(c1)

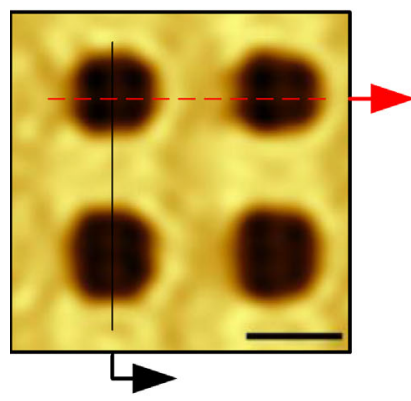

(d1)

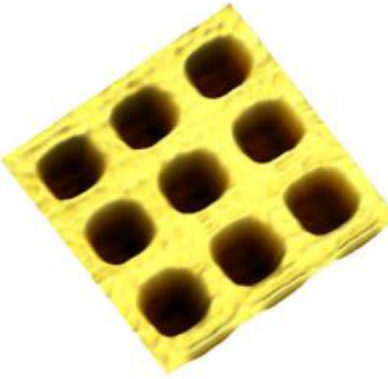

(a2)

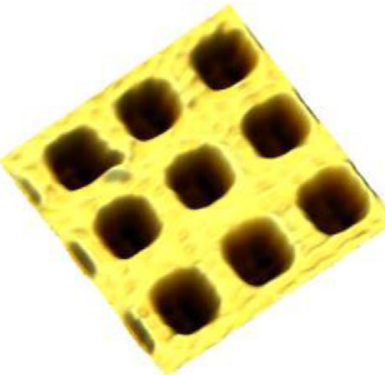

(b2)

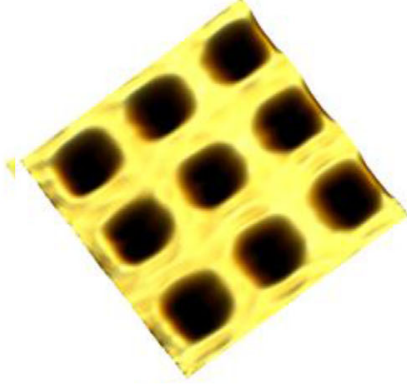

(c2)

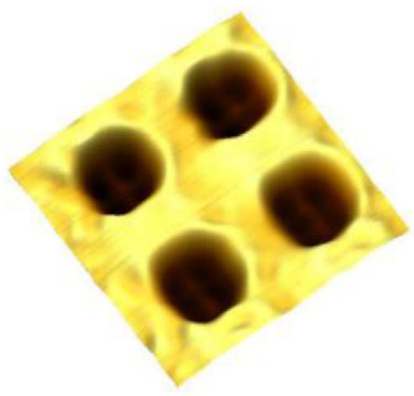

(d2)

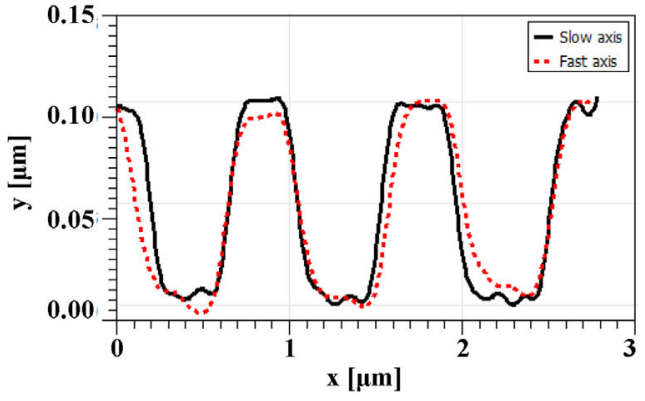

(a3)

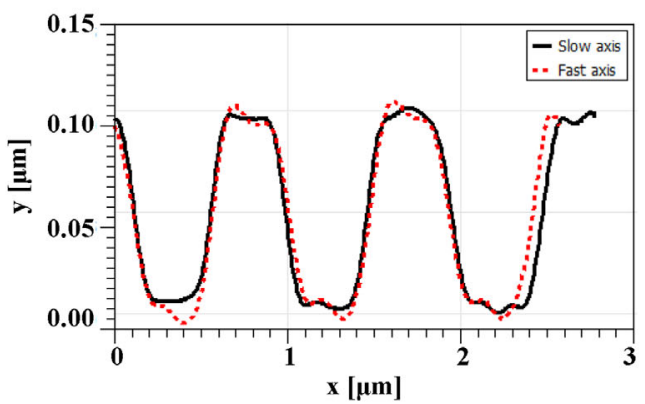

(b3)

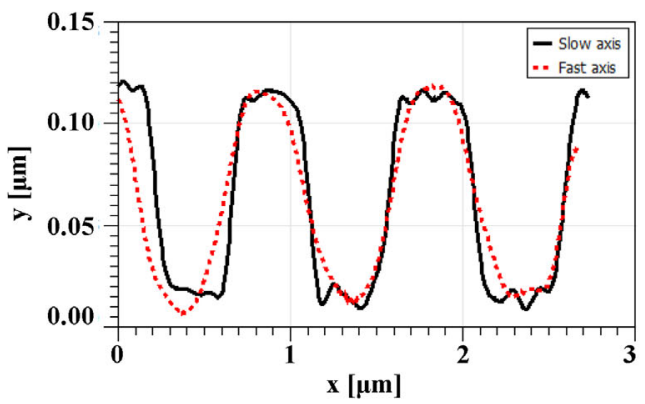

(c3)

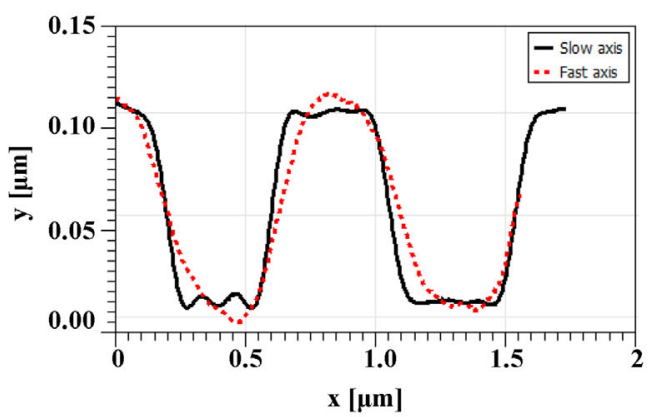

(d3)

Fig 9. Scanned images, 3D renderings and line traces obtained at $256 \mathrm{~Hz}(\mathrm{a} 1)-(\mathrm{a} 3), 512 \mathrm{~Hz}$ (b1)-(b3), 768Hz(c1)-(c3), and 1024 Hz (d1)-(d3), respectively. The scale bar of the images is. All of AFM images were processed using Gwyddion. Firstly, the bow shape background was removed by implementing polynomial fitting, then using 2D FFT filter to eliminate the noise.

point series contained information of one period were constructed and sent to the DAQ card, the function of which was to reproduce such one period several times per second. The displacement of the X-scanner with (black line) and without (red line) hysteresis compensation are shown in Figure 8(b). It is clear that the nonlinearity of hysteresis has been reduced considerably. From the images presented by Figure 8(e), the shapes of the standard grating were much more square after being compensated (compared to Figure 8(d) where the compensation was not implemented), meaning that scanner displaced almost linearly in both fast 
and slow axes. Additionally, we also confirm that at the given driving frequency the hysteresis of piezoactuator is independent with the scan range (Watanabe et al., 2013), which simplified the process of hysteresis linearization significantly.

\section{Imaging Results}

Performance of the presented high speed tripod scanner was evaluated by varying the scan rates from 256 to $1024 \mathrm{~Hz}$, operating in constant-force contact mode. The scanner's stand-alone nature makes sample preparation and mounting practically easy, without being affected by sample's weight or volume. Figure 9 shows the obtained images, 3D renderings and singletrace lines. The scan rates were set at 256, 512, 768, and $1024 \mathrm{~Hz}$, respectively, corresponding to imaging rates from $1 \mathrm{frame} / \mathrm{s}$ up to $4 \mathrm{frame} / \mathrm{s}$. Under these conditions, the maximum tip speed reached $4.1 \mathrm{~mm} / \mathrm{s}$.

It is apparent that the lateral dynamics of the scanner were well compensated so that the images were not distorted by vibration-introduced ripples which were commonly seen when resonance peaks in the transfer function still existed. The black lines shown in crosssection curves were acquired from the slow axis direction, while red lines represented the measured data of fast axis, as indicated in the 2D images. From the single-trace curves one can see that as the scan rate increases, the edges of the step became rounded due to the limitation of Z-axis feedback loop bandwidth, especially when the scan rates reached $1024 \mathrm{~Hz}$, therefore the scan range was reduced to have a better resolution. Images with scan rates higher than $1024 \mathrm{~Hz}$ were not recorded because the quality of the images already started to deteriorate. This problem mainly arises from the limited mechanical bandwidth, more specifically, the use of a large piezoactuator (since a larger travel rage of $\mathrm{Z}$-scanner is preferred for practical use) make the bandwidth of Z-axis far below the bandwidth of other components in the feedback loop. Moreover, another possible reason is the heavy load introduced by the piezo's large capacitance, which slows down the response of the high voltage amplifier. Therefore, by designing stiffer planar flexure or changing to a more compact piezoactuator, the mechanical bandwidth of the Z-scanner could be effectively enlarged.

\section{Conclusions}

In this paper, a high speed tip-scanning AFM is presented based on a tripod-type scanner and selfsensing cantilevers. Carefully designed planar flexures are used to guide the motion of piezoactuator and strengthen its lateral stiffness. As a result, the first resonance mode of the $\mathrm{X}-, \mathrm{Y}-$, and $\mathrm{Z}$-axes reaches
$24.5,22.3$, and $26.7 \mathrm{kHz}$, with the travel range of 5.8 , 6.0 , and $5.9 \mu \mathrm{m}$, respectively. To evaluate the performance of our home-built high speed AFM system, AFM images are acquired at scan rates up to $1 \mathrm{kHz}$. However, higher scan rates are not achieved because of the limitation of the Z-axis mechanical bandwidth. In future work, we plan to further improve the current design, aiming to achieve high-quality video-rate $\mathrm{AFM}$ imaging.

\section{Acknowledgments}

C.Y. acknowledges the financial support from the China Scholarship Council for his joint PhD project (Grant No.201306120115). The authors would also like to thank S.A for his help with the test of piezo amplifier bandwidth. Author contributions: C.Y. conceived the experiments, designed and built the instrument, performed the experiments and accomplished data analysis. J.Y. supervised the project and performed data analysis. M.D. and N.H. designed the instrument. J.Z. supervised the project. G.E.F conceived the experiments, and supervised the project. All of authors contributed to the writing of the paper.

\section{References}

Adams JD, Erickson BW, Grossenbacher J, et al. 2015. Harnessing the damping properties of materials for high-speed atomic force microscopy. Nat Nanotechnol 11:147-151.

Ando T, Uchihashi T, Fukuma T. 2008. High-speed atomic force microscopy for nano-visualization of dynamic bimolecular processes. Prog Surf Sci 83:337-437.

Braunsmann C, Schäffer TE. 2010. High-speed atomic force microscopy for large scan sizes using small cantilevers. Nanotechnology 21:225705.

Casuso I, Khao J, Chami M, et al. 2012. Characterization of the motion of membrane proteins using high-speed atomic force microscopy. Nat Nanotechnol 7:525-529.

Croft D, Shed G, Devasia S. 1999. Creep, hysteresis, and vibration compensation for piezoactuators: atomic force microscopy application. J Dyn Sys Meas Control 123:35-43.

Fantner GE, Hegarty P, Kindt JH, et al. 2005. Data acquisition system for high speed atomic force microscopy. Rev Sci Instrum 76:026118.

Fantner GE, Schitter G, Kindt J, et al. 2006. Components for high speed atomic force microscopy. Ultramicroscopy 106:881-887.

Fantner GE, Barbero RJ, Gray DS, et al. 2010. Kinetics of antimicrobial peptide activity measured on individual bacterial cells using high-speed atomic force microscopy. Nat Nanotechnol 5:280-285.

Fleming AJ, Leang KK. 2008. Charge drives for scanning probe microscope positioning stages. Ultramicroscopy 108:1551-1557.

Fleming AJ. 2009. A megahertz bandwidth dual amplifier for driving piezoelectric actuators and other highly capacitive loads. Rev Sci Instrum 80:104701.

Fukuma T, Okazaki Y, Kodera N, et al. 2008. High resonance frequency force microscope scanner using inertia balance support. Appl Phys Lett 92:243119-243121.

Hansma PK, Cleveland JP, Radmacher M, et al. 1994. Tapping mode atomic force microscopy in liquids. Appl Phys Lett 64:1738-1740. 
Humphris ADL, Miles MJ, Hobbs JK. 2005. A mechanical microscope: high-speed atomic force microscopy. Appl Phys Lett 86:34106.

Itani T, Santillan JJ. 2010. In situ characterization of photoresist dissolution. Appl Phys Express 3:061601.

Kenton BJ, Leang KK. 2012. Design and control of a three-axis serial-kinematic high-bandwidth nanopositioner. IEEEASME T Mech 17:356-369.

Kindt JH, Fantner GE, Cutroni JA, et al. 2004. Rigid design of fast scanning probe microscopes using finite element analysis. Ultramicroscopy 100:259-265.

Kodera N, Sakashita M, Ando T. 2006. Dynamic proportionalintegral-differential controller for high-speed atomic force microscopy. Rev Sci Instrum 77:083704.

Kodera N, Yamamoto D, Ishikawa R, et al. 2010. Video imaging of walking myosin $\mathrm{V}$ by high-speed atomic force microscopy. Nature 468:72-76.

Kuiper S, Schitter G. 2012. Model-based feedback controller design for dual actuated atomic force microscopy. Mechatronics 22:327-337.

Leang KK, Devasia S. 2007. Feedback-linearized inverse feedforward for creep, hysteresis, and vibration compensation in AFM piezoactuators. IEEE T Contr Syst T 15: 927-935.

Mahmood IA, Moheimani SOR. 2009. Fast spiral-scan atomic force microscopy. Nanotechnology 20:365503.

Nievergelt AP, Erickson BW, Hosseini N, et al. 2015. Studying biological membranes with extended range high-speed atomic force microscopy. Sci Rep 5:11987.
Schitter G, Åström KJ, DeMartini BE, et al. 2007. Design and modeling of a high-speed AFM-scanner. IEEE T Contr Syst T 15:906-915.

Schitter G, Phan N. 2008. Field programmable analog array (FPAA) based control of an atomic force microscope. IEEE American Control Conference 2008:2690-2695.

Schitter G, Thurner PJ, Hansma PK. 2008. Design and inputshaping control of a novel scanner for high-speed atomic force microscopy. Mechatronics 18:282-288.

Sulchek T, Hsieh R, Adams JD, et al. 2000. High-speed tapping mode imaging with active $\mathrm{Q}$ control for atomic force microscopy. Appl Phys Lett 76:1473-1475.

Sulchek T, Yaralioglu GG, Quate CF, et al. 2002. Characterization and optimization of scan speed for tapping-mode atomic force microscopy. Rev Sci Instrum 73:2928-2936.

Tuma T, Lygeros J, Kartik V, et al. 2012. High-speed multiresolution scanning probe microscopy based on Lissajous scan trajectories. Nanotechnology 23:185501.

Watanabe H, Uchihashi T, Kobashi T, et al. 2013. Wide-area scanner for high-speed atomic force microscopy. Rev Sci Instrum 84:053702.

Yong YK, Moheimani SOR, Petersen IR. 2010. High-speed cycloidscan atomic force microscopy. Nanotechnology 21:365503.

Yong YK, Moheimani SOR, Kenton BJ, et al. 2012. High-speed flexure-guided nanopositioning: mechanical design and control issues. Rev Sci Instrum 83:121101.

Yong YK, Bhikkaji B, Moheimani SOR. 2013. Design, modeling, and FPAA-based control of a high-speed atomic force microscope nanopositioner. IEEE-ASME T Mech 18:1060-1071. 